\title{
High Resolution Imaging and X-Ray Microanalysis at High Count Rate: The Supreme Achievement in Materials Characterization
}

\author{
Raynald Gauvin ${ }^{1}$, David C. Joy ${ }^{2,3}$, Nicolas Brodusch ${ }^{1}$, Hendrix Demers ${ }^{1}$ and Patrick Woo ${ }^{4}$ \\ 1 Department of Materials Engineering, McGill University, Montréal, QC, Canada. \\ 2 University of Tennessee, Electron Microscopy Facility, Knoxville, TN, USA. \\ 3 Oak Ridge National Lab, Centre NanoPhase Materials Science, Oak Ridge, TN, USA. \\ ${ }^{4}$ Hitachi High-Tech Canada, Rexdale, ON, Canada.
}

The scanning electron microscope (SEM) was developed for imaging applications and later, with the introduction of the $\mathrm{Si}(\mathrm{Li})$ EDS detector, simultaneous imaging and $\mathrm{x}$-ray microanalysis became possible. However, long working distances were needed to use the EDS detector because the SEM was initially optimized for imaging only where the best spatial resolution is generally obtained at short working distance. This problem is still topical today and unfortunately x-ray microanalysis is never performed in the best imaging conditions, i.e., not with the smallest probe size. With the introduction of the Bruker QUANTAX Annular QUAD silicon drift detector (SDD) system, scanning electron microscopy is facing a revolution. Since this detector is inserted below the pole piece, a low working distance, which gives a high solid angle up to 1.2 sr, can be used and an improved spatial resolution becomes possible. Also, since the count rate can be as high as $2000000 \mathrm{cps}$, many counts will be acquired in a short period of time, allowing to lower significantly the detection limit of elements and as well the minimum size of different phase features. At this point, the time required for $\mathrm{x}$-ray imaging will be of the same order as for the atomic number contrast images achieved through backscattered electrons (BSE) imaging [1]. With this X-ray detector installed on a cold field emission scanning electron microscope, like the HITACHI SU-8020, quantitative x-ray microanalysis with high spatial resolution at low beam energy becomes possible with the possibility of using the various imaging modes of the top and upper detector at the same time.

Figure 1 shows a BSE image and $\mathrm{x}$-ray maps of $\mathrm{BaCO}_{3}$ and $\mathrm{TiO}_{2}$ powder mixture analysed at 3 $\mathrm{keV}$ with the Bruker Annular QUAD SDD detector in a HITACHI SU-8020 SEM with 3 minute acquisition time. The high resolution details in the $\mathrm{x}$-ray maps are truly impressive with such a short acquisition time. The smallest particulates are around $40 \mathrm{~nm}$. Figure 2 shows a secondary electron (SE) image and x-ray maps of a Li based material for batteries applications analysed in similar conditions. Again, the spatial resolution obtained was impressive and the smallest particulate observed was around $35 \mathrm{~nm}$. This paper will present many examples of high resolution x-ray microanalysis and imaging at low electron energy with high count rates as well as in the scanning transmitted mode at $30 \mathrm{keV}$ on thin specimens [2].

\section{References:}

[1] D.E. Newbury and N.W.M. Ritchie, Scanning, 33 (2011), pp. 174-192.

[2] N. Brodusch, M. Trudeau, P. Michaud, L. Rodrigue, J. Boselli and R. Gauvin, Microscopy and Microanalysis, 18 (2012), pp. 1393-1409. 

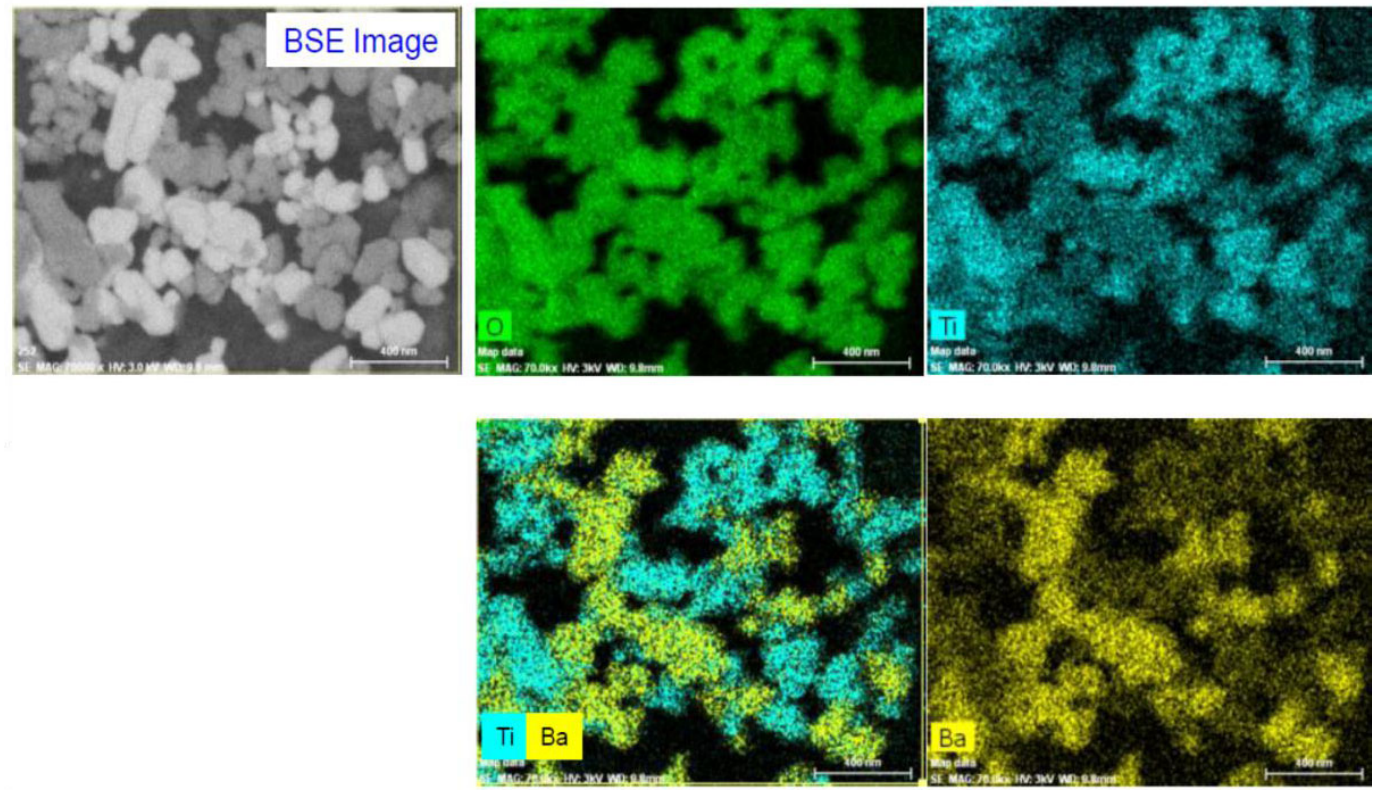

Figure 1: $\mathrm{BSE}$ image and $\mathrm{x}$-ray maps of $\mathrm{BaCO}_{3}$ and $\mathrm{TiO}_{2}$ powder mixture analysed at an accelerating voltage of $3 \mathrm{keV}$ and a probe current of $180 \mathrm{pA}$ with the Bruker Annual QUAD SDD detector in a HITACHI SU-8020 SEM without beam drift correction. The acquisition time was 3 minutes.
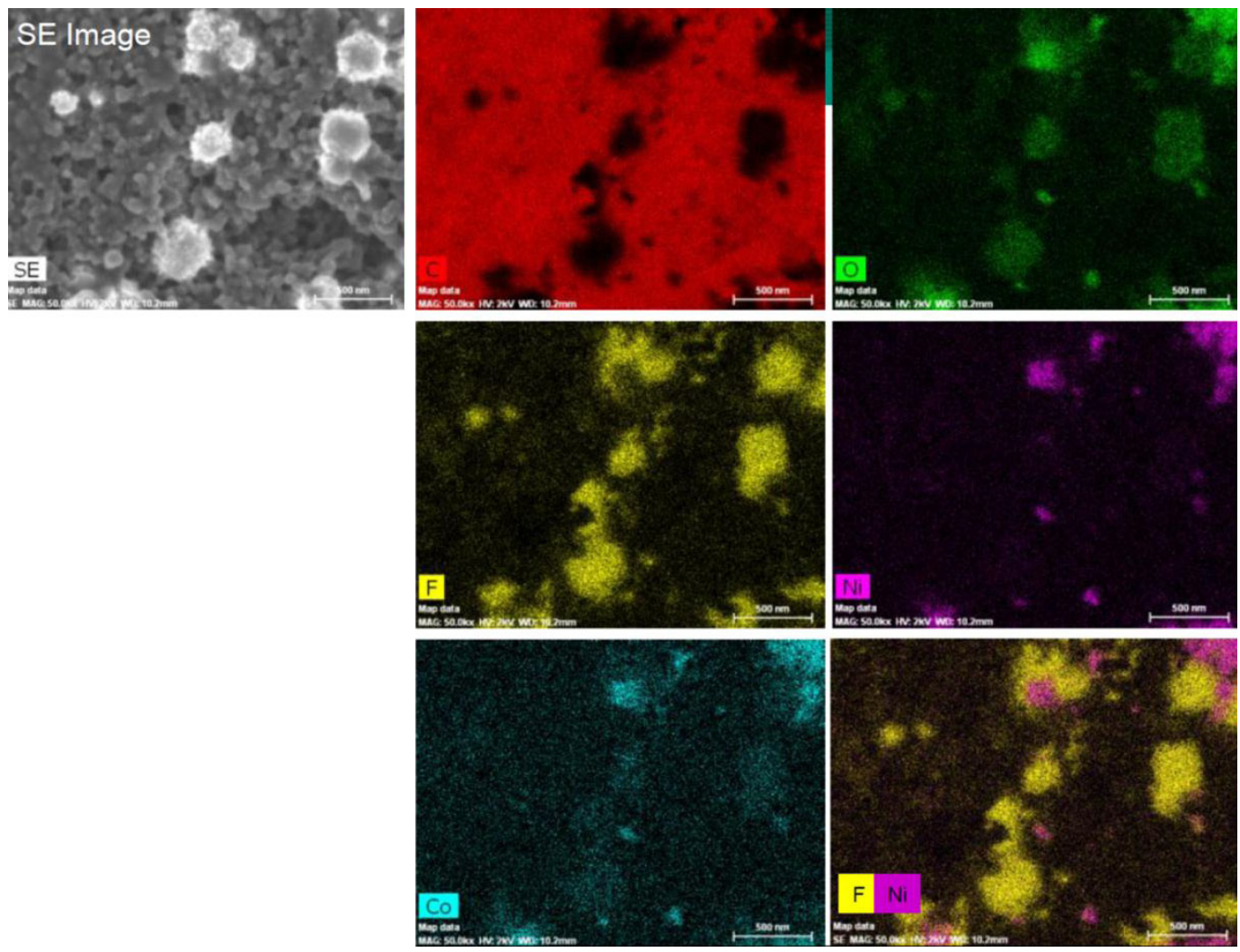

Figure 2: SE image and x-ray maps of a $\mathrm{Li}$ based material for batteries applications analysed at an accelerating voltage of $2 \mathrm{keV}$ and a probe current of $260 \mathrm{pA}$ with the Bruker Annual QUAD SDD detector in a HITACHI SU-8020 without beam drift correction. The acquisition time was 3 minutes. 